\title{
Emergency Departments in the Detection of COVID-19 Cases; Multi-Centered Data From Turkey
}

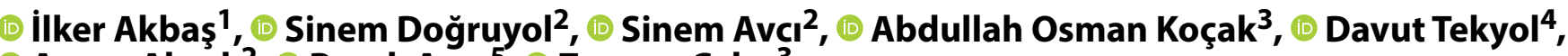 \\ Aycan Akcalı², Burak Acar ${ }^{5}$, (i) Zeynep Çakır ${ }^{3}$ \\ ${ }^{1}$ Department of Emergency Medicine, Bingol State Hospital, Bingol, Turkey \\ ${ }^{2}$ Department of Emergency Medicine, Manisa Merkez Efendi State Hospital, Manisa, Turkey \\ ${ }^{3}$ Department of Emergency Medicine, Ataturk University Faculty of Medicine, Erzurum, Turkey \\ ${ }^{4}$ Department of Emergency Medicine, Haydarpasa Numune Training and Research Hospital, Istanbul, Turkey \\ ${ }^{5}$ Department of Emergency Medicine, Katip Celebi University, Ataturk Training and Research Hospital, Izmir, Turkey
}

\begin{abstract}
Introduction: The aim of this study is to examine the story, clinical findings, initial computed tomography (CT) findings, and reverse transcription-polymerase chain reaction (RT-PCR) results and to evaluate the consistency of this data.

Methods: This is a multi-center retrospective study and out of patients aged 18 and over who were evaluated for Coronavirus disease 2019 (COVID-19) in the emergency department between April 01, 2020 and May 01, 2020. Data regarding the patients such as age, gender, comorbidity, COVID-19 contact, symptoms, vital findings, laboratory parameters, CT findings, hospitalization status, and case fatality rate (CFR) were examined.

Results: About $62.4 \%$ of 687 patients were male, the mean age was 49.7. About $49.9 \%$ of patients had at least one comorbidity. The most common symptoms were fever (61.6\%), cough (56.5\%), and dyspnea (33.2\%). About 33.9\% of patients had RTPCR positivity, $69.9 \%$ had CT positivity, and $72.5 \%$ had both RT-PCR and CT positivity. Sensitivity of CT was 72.5 , its specificity was $31.5 \%$, and its accuracy was $45.5 \%$. Most common CT pattern was pure GGO (47.9\%). CFR was $6 \%$ and it was significantly higher in both RT-PCR and CT positive patients $(p=0.01)$. According to logistic regression analysis, male gender [ $p=0.037$; odds ratio (OR): 0.385; 95\% confidence interval (Cl), 0.157-0.943], higher age ( $p=0.000 ;$ OR: 1.068; 95\% Cl, 1.031-1.106), and comorbidity presence ( $p=0.008$; OR: $5.374 ; 95 \% \mathrm{Cl}, 1.539-7.618$ ) were found to be associated with mortality.

Discussion and Conclusion: In our study, we found that using RT-PCR and CT together and supporting them with clinical data were the strongest approach in the diagnosis of COVID-19 cases.

Keywords: Computed tomography; COVID-19; Coronavirus; diagnosis; emergency medicine; mortality; 2019-nCoV.
\end{abstract}

Se evere acute respiratory syndrome coronavirus 2 which causes severe respiratory syndrome, was first identified in Wuhan, China, and spread around the world, becoming a global public health problem ${ }^{[1]}$. When Coronavirus diease 2019 (COVID-19) Pandemic was declared by the World
Health Organization (WHO) in January 2020, the infection had reached 24 countries and five continents internationalIy ${ }^{[2]}$. In Turkey, the total number of COVID-19 cases by May 14,2020 , was 143.114 and the total number of deaths from pandemic was declared as $3952^{[3]}$. 
In the definitive diagnosis of COVID-19, reverse transcription-polymerase chain reaction (RT-PCR) is used as a reference test ${ }^{[4]}$. However, thoracic computed tomography (CT) has been used as an important early diagnostic tool after being accepted in early detection of cases and determining the severity of the disease ${ }^{[5]}$. The most common pathological finding in studies where patient CTs are examined in COVID-19 pneumonia; is the existence of bilateral, multifocal, and peripheral ground glass opacities (GGO) ${ }^{[6-8]}$. These imaging characteristics are considered to be the main findings that bring COVID-19 infection to mind, but they must be validated with positive RT-PCR results for definitive diagnosis ${ }^{[9]}$.

No specific treatment or vaccine is available for COVID-19 yet ${ }^{[10]}$. Therefore, rapid detection and isolation of cases are the most important step that can affect the spread process of the infection. In the process of COVID-19 Pandemic, the most challenging aspect for clinicians was the inability to be diagnosed with a single laboratory parameter or radiological imaging method. Our aim in this study was to examine the consistency of clinical findings, initial CT findings and RT-PCR results in possible/definitive COVID-19 cases. For this purpose, we evaluated in detail the compliance of the findings obtained during the clinical evaluation process, which began from the moment our patients first stepped into the emergency department, with diagnosis and imaging tests.

\section{Materials and Methods}

\section{Study Design}

This study is a multi-centered study with a retrospective design. The study was conducted in accordance with the principles of the Declaration of Helsinki. The ethics committee approval for the study was taken from the ethics committee of Atatürk University Faculty of Medicine (decision number: 75). Data on patient applications between April 1,2020, and May 1, 2020, were obtained from the hospital data system.

\section{Patient Population and Clinical Data}

The study included possible and definitive COVID-19 cases chosen among patients aged 18 and over who were evaluated in the emergency department during the COVID-19 pandemic process, as specified in the algorithm of the Ministry of Health of the Republic of Turkey ${ }^{[11]}$.

The study was organized in two different provinces, in two centers. One of the centers, Atatürk University Faculty of Medicine is a "pandemic hospital" and the emergency department has functioned normally in addition to admitting
COVID-19 cases. The other center, Merkezefendi State Hospital, functioned as a "quarantine hospital," and no other patient admissions were made to the emergency department except for possible and definitive COVID-19 cases. Patients applying as outpatients in both centers were evaluated and examined in accordance with the triage algorithm of our ministry (Appendix 1). Laboratory examinations of all patients in the study and unenhanced thoracic CT prompts were planned according to the algorithm (Appendix 2). Within $24 \mathrm{~h}$ of patient applications in both centers, the first RT-PCR test and the first CT imaging were performed. The second RT-PCR test was performed at least $48 \mathrm{~h}$ after the first. For patients with multiple RT-PCR assays, the diagnosis of COVID-19 was confirmed when any one of the nucleic acid test results was positive. To be included in the study:

1. Patient who meet the definition of possible/definitive case in terms of clinical, story, and physical examination findings

2. Patient with positive COVID-19 CT

3. Patient whose first RT-PCR result is positive and comes with referral.

Patients who had met at least one of these three conditions were included in the study. Patients who do not comply with the possible and/or definitive COVID-19 case definition, those who do not have CT imaging made within $24 \mathrm{~h}$ of application, and patients with missing data in their files were excluded from the study.

Age, gender, comorbidity, admission and contact information of patients; symptoms, vital findings, laboratory parameters, service/intensive care hospitalization status, hospital stay time, and case fatality rate (CFR) were retrospectively examined. Symptoms of fever, cough, and dyspnea were evaluated as "alarm symptoms." Intensive care hospitalization, mortality, and increased hospital stay times were considered "poor outcome."

\section{CT Image Data Acquisition and Analysis}

For the study, axial CT sections with a range of $5 \mathrm{~mm}$ were evaluated. All images were viewed on both lung (width, $1500 \mathrm{HU}$; level, $-700 \mathrm{HU}$ ) and mediastinal (width, $350 \mathrm{HU}$; level, $40 \mathrm{HU}$ ) settings. CT images of patients were evaluated together by two emergency medical professionals with at least 10 years of experience, who knew the clinical data of patients but were blind about RT-PCR results. These results were compared with radiology reports in the hospital system. Accordingly, patients were evaluated in two groups as CT positive and CT negative. In addition, the CT findings detected were recorded in detail. Kappa analysis was per- 


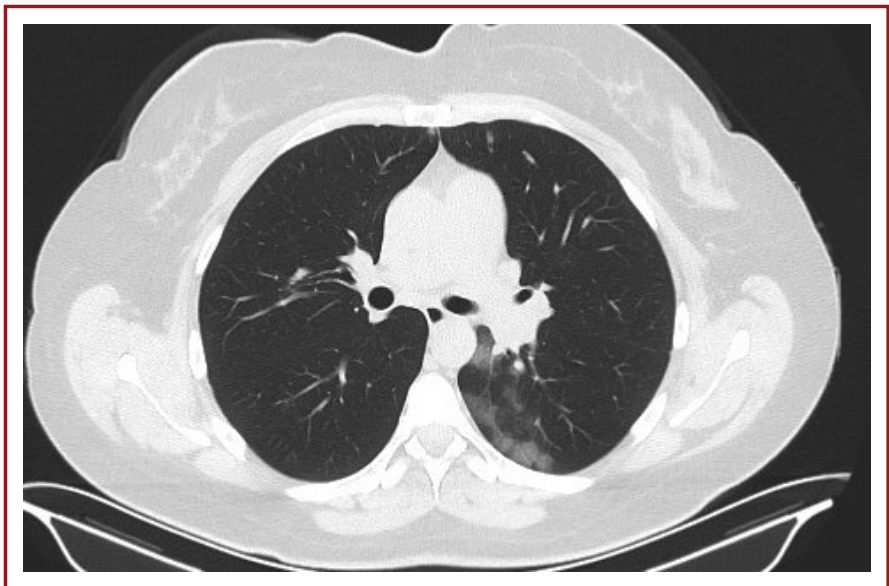

Figure 1. A 30-year-old woman who had a positive contact history but no symptoms. She had a positive initial reverse transcriptionpolymerase chain reaction result. Axial computed tomography image shows unilateral multifocal lesions with pure ground-glass opacities in a rounded morphology.

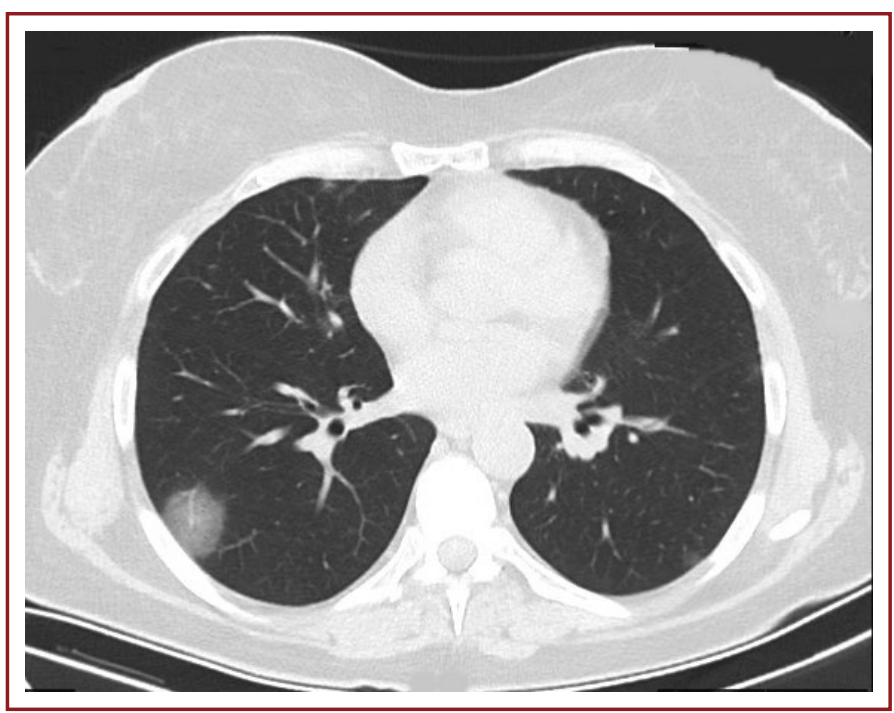

Figure 3. A 52-year-old woman who had cough and dyspnea and had positive initial reverse transcription-polymerase chain reaction result. Axial computed tomography image shows a rounded groundglass opacity with subpleural distribution and thickened interlobular septa, presenting as typical crazy paving pattern (arrow).

formed for the correlation of CT reports between radiology reporting service and emergency medicine specialists.

The review of CT findings associated with COVID-19 lung involvement was conducted using four different categories ${ }^{[12]}$.

\section{Lung involvement: Right/left/bilateral}

2. Extent of lesions; focal, multifocal, diffuse

3. Pattern of opacities;

- Pure GGO pattern (ground glass appearance with a clear view of pulmonary vessels) (Fig. 1).

- Complicated GGO pattern (GGO with reticular and/or

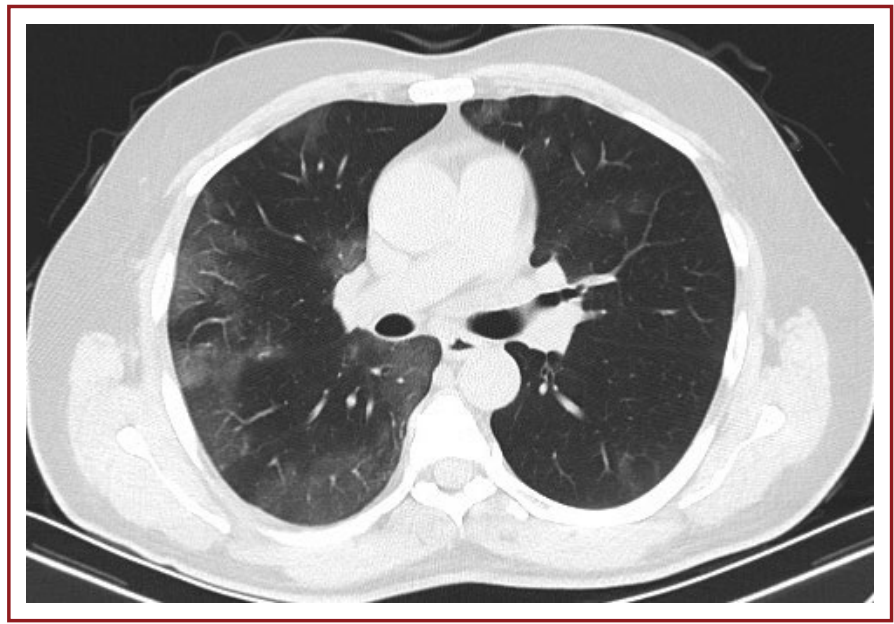

Figure 2. A 51-year-old man who had fever and cough and a positive initial reverse transcription-polymerase chain reaction result. Axial computed tomography image shows multifocal, peripheral, patchy, and band-like ground-glass opacities together.

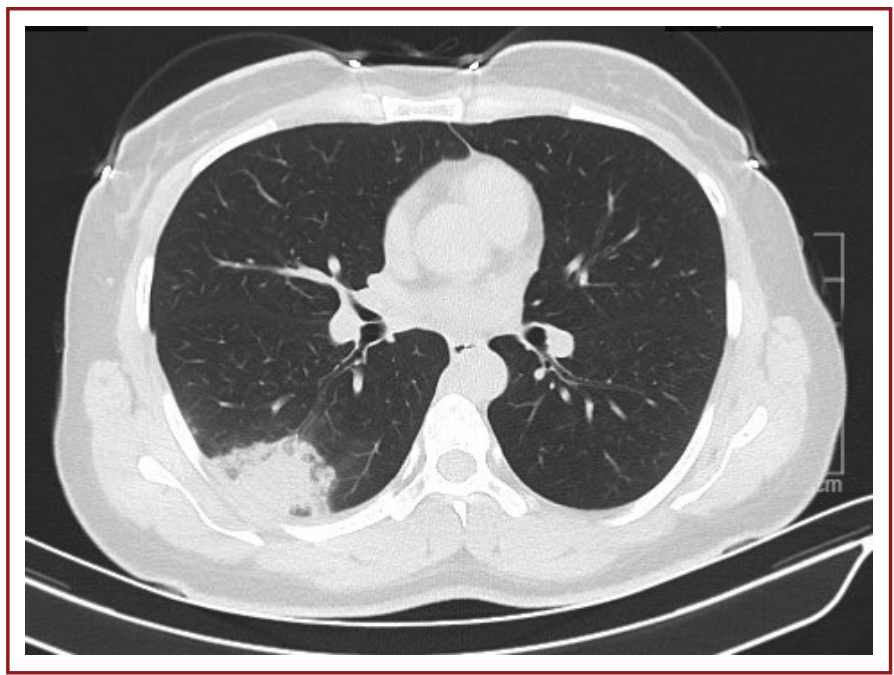

Figure 4. A 66-year-old woman had fever, cough, and dyspnea and had multiple positive reverse transcription-polymerase chain reaction results. Axial computed tomography image shows unilateral focal pulmonary consolidation.

interlobular septal thickening pattern) (Fig. 2, 3).

- Consolidation pattern (the presence of homogeneous opacities where the vessels are obliterated) (Fig. 4).

- Mixed pattern [a combination of consolidation, (GGO), and reticular opacities] (Fig. 5).

4. Accompanying radiological finding; the presence of pleural effusion, atelectasis, nodule, lymphadenopathy, (defined as lymph node size $\geq 10 \mathrm{~mm}$ in short-axis dimension).

A subgroup was created for cases with pure GGO and complicated GGO pattern while examining lesion patterns. This subgroup was described as "GGO-related pattern." 


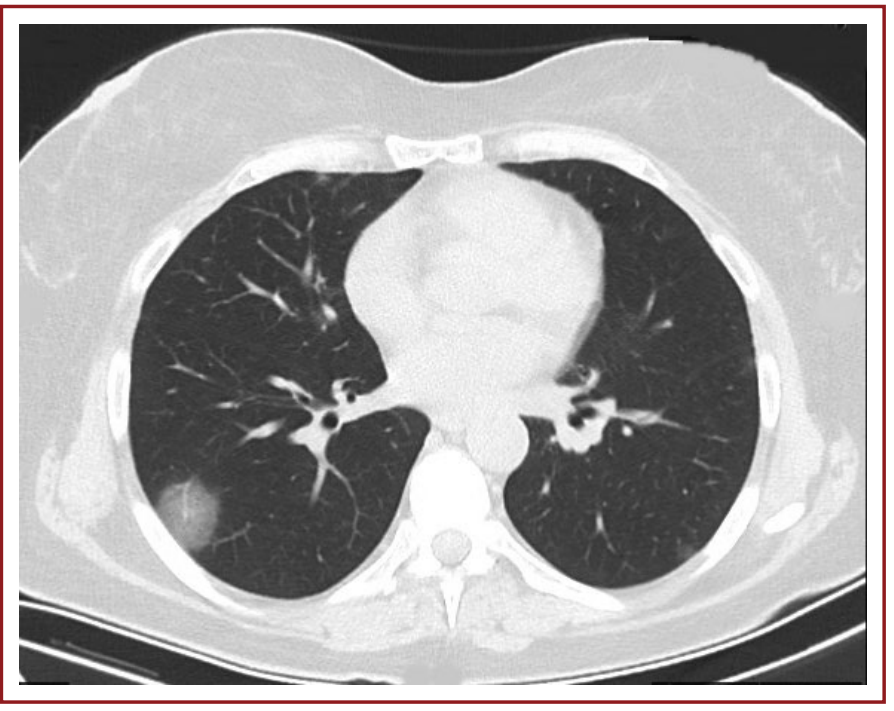

Figure 5. A 42-year-old man who had cough and dyspnea and had positive reverse transcription-polymerase chain reaction results. Axial computed tomography image shows multiple bilateral consolidations with ground-glass opacities in subpleural region of the lower lobes (mixed pattern).

\section{Statistical Analysis}

All statistical analyses were performed in IBM SPSS Statistics forWindows, Version 20.0 (IBM Corp., Armonk, NY, USA). Normal distribution was tested with the Kolmogorov-Smirnov test. Percentages and frequencies for categorical variables and mean ( \pm standard deviation) values for continuous variables were determined. The Student's t-test was used for two continuous group comparisons. The Pearson's X2test was used for categorical variables. The inter-observer agreement was analyzed by kappa analysis. By considering RT-PCR results as a reference for COVID-19 diagnosis, sensitivity, specificity, accuracy, positive predictive value (PPV), and negative predictive value (NPV) for CT were calculated. About $95 \%$ confidence interval $(\mathrm{Cl})$ for these values was provided by the Wilson score method. With logistic regression analysis, the arguments that can be effective on mortality termination were examined and odds ratio (OR) data were specified. $\mathrm{P}=0.05$ was considered significant.

\section{Results}

\section{Patient Characteristics}

Of the 687 patients included in the study, $429(62.4 \%)$ male and $258(37.6 \%)$ women. The average age was $49.7 \pm 19.1$ (range 18-94). The number of patients with at least one comorbid disease was 343 (49.9\%). The most frequent comorbid diseases were listed as hypertension (28.8\%) and chronic pulmonary diseases (16.3\%). There was no signif- icant difference between patients in the study for RT-PCR positivity and $C T$ positivity ( $p>0.05$ ). No significant relationship was detected between the presence of comorbid disease and RT-PCR positivity ( $p>0.05$ ). CT positivity was higher in those with comorbid disease compared to other patients $(p=0.001)$. In addition, length of stay in hospital for those with comorbid disease had increased significantly (mean difference: $-0.94,95 \% \mathrm{Cl}:-1.56,-0.29$ ) $(p=0.004)$. Patient age, gender, and comorbidity data are summarized in Table 1.

\section{Symptoms}

646 of all patients (94\%) had at least one symptom. The most common symptoms were high fever (61.6\%), cough (56.5\%), and dyspnea (33.2\%). The relationship between fever, cough and dyspnea, and diagnostic tests is shown in Table 2. The most common alarm symptoms were fever for 56 patients with only positive RT-PCR among 646 patients with symptoms (51.8\%). Among these patients with symptoms, coughing was the most common alarm symptom for patients with only CT positive (67.6\%). The most common alarm symptom among 157 patients with symptoms having both RT-PCR and CT positive results was fever (67.5\%). Only 41 (6\%) of patients in the study had no symptoms. In 27 of these, 41 patients (65.9\%) CT positivity and in 20 patients (48.8\%) PCR positivity were present.

\section{Vital Signs}

The vital findings of the patients are shown in Table 1. The average fever measured in patients with symptoms compared to those with no symptoms was measured to be highly significant (mean difference: $-0.55,95 \% \mathrm{Cl}:-0.73$, $-0.36)(p=0.000)$. Oxygen saturation values were also significantly lower in patients with any symptom (mean difference: $1.76,95 \% \mathrm{Cl}: 1.13,2.38)(\mathrm{p}=0.000)$. CT positive patients' saturation values were significantly lower (mean difference: $1.53,95 \% \mathrm{Cl}: 0.92,2.14)(\mathrm{p}=0.000)$.

\section{Diagnostic Procedures}

High level of concordance between the researchers who evaluated the patient's radiologic reports was found to be $(k=0.92)(p<0.001)$. When the RT-PCR results of the patients were examined, 233 were positive and 454 were negative, and the RT-PCR positivity rate was $33.9 \%$ ( $95 \% \mathrm{Cl}$ : $30-37 \%)$. Thoracic CT results were positive in 480 patients and negative in 207 patients and the CT positivity rate was $69.9 \%$ (95\% Cl: $66-73 \%)$. Among RT-PCR positive patients, the rate of patients with positive CT was 169/233 (72.5\%) and 157 of the 169 patients with both RT-PCR and CT positive 
Table 1. Patient characteristics and laboratory results

\begin{tabular}{|c|c|c|c|}
\hline Parameters & All patients & Both RT-PCR and CT $(+)$ patients $(n=169)$ & $\mathbf{p}$ \\
\hline Male & $429(62.4)$ & $97(57.4)$ & 0.119 \\
\hline Age, $\mathrm{n}(\%)$ & $49.7 \pm 19.1$ & $53.2 \pm 16.7$ & 0.003 \\
\hline \multicolumn{4}{|l|}{ Admission type } \\
\hline Direct application & $43(6.3)$ & $3(1.8)$ & 0.006 \\
\hline Positive contact history & $109(15.9)$ & $49(29.0)$ & 0.000 \\
\hline \multicolumn{4}{|l|}{ Comorbidities, n (\%) } \\
\hline Any & $344(50.1)$ & $89(52.7)$ & 0.413 \\
\hline Hypertension & $198(28.8)$ & $57(33.7)$ & 0.105 \\
\hline Cerebrovascular diseases & $31(4.5)$ & $5(3.0)$ & 0.262 \\
\hline Chronic kidney disease & $22(3.2)$ & $2(1.2)$ & 0.086 \\
\hline Other & $47(6.8)$ & $16(9.5)$ & 0.119 \\
\hline Duration of symptoms, day & $2.5 \pm 1.0$ & $2.6 \pm 0.9$ & 0.366 \\
\hline \multicolumn{4}{|l|}{ Symptoms, n (\%) } \\
\hline Any & $646(94.0)$ & $157(92.9)$ & 0.474 \\
\hline Fever & $420(61.1)$ & $106(65.4)$ & 0.439 \\
\hline Cough & $388(56.5)$ & $84(51.9)$ & 0.065 \\
\hline Dyspnea & $228(33.2)$ & $55(34.0)$ & 0.955 \\
\hline Myalgia & $105(15.3)$ & $28(17.6)$ & 0.495 \\
\hline \multicolumn{4}{|l|}{ Vital signs } \\
\hline Body temperature, ${ }^{\circ} \mathrm{C}$ & $37.4 \pm 1.0$ & $37.3 \pm 0.9$ & 0.008 \\
\hline Systolic blood pressure, $\mathrm{mmHg}$ & $132 \pm 22$ & $130 \pm 21$ & 0.088 \\
\hline Diastolic blood pressure, $\mathrm{mmHg}$ & $80 \pm 12$ & $80 \pm 11$ & 0.562 \\
\hline Heart rate, bpm & $92 \pm 16$ & $89 \pm 15$ & 0.003 \\
\hline Oxygen saturation, $\%$ & $96 \pm 4$ & $95 \pm 6$ & 0.008 \\
\hline \multicolumn{4}{|l|}{ Laboratory results } \\
\hline Leukocyte count ( $\times 10^{9} / \mathrm{L}$; normal range $\left.4-10\right)$ & $9.2 \pm 4.1$ & $7.3 \pm 3.5$ & 0.000 \\
\hline Neutrophil count $\left(\times 10^{9} / \mathrm{L}\right.$; normal range $\left.2-7\right)$ & $6.8 \pm 3.9$ & $5.2 \pm 3.3$ & 0.000 \\
\hline Lymphocyte count ( $\times 10^{9} / \mathrm{L}$; normal range $\left.0.8-4\right)$ & $1.7 \pm 0.9$ & $1.5 \pm 0.7$ & 0.000 \\
\hline Platelet count $\left(\times 10^{9} / \mathrm{L}\right.$; normal range $\left.150-450\right)$ & $227.25 \pm 77.42$ & $217.82 \pm 70.45$ & 0.036 \\
\hline Hemoglobin level (g/L; normal range 11.7-15.5) & $13.4 \pm 2.2$ & $13.8 \pm 2.0$ & 0.096 \\
\hline Procalcitonin (ng/mL; normal range $0-0.1)$ & 0.01 (IQR: 0.09) & 0.1 (IQR: 0.09) & 0.755 \\
\hline D-dimer (ng/mL; normal range 0-150) & 150 (IQR: 162) & 150 (IQR: 0.116) & 0.102 \\
\hline Troponin I (ng/mL; normal range 0-0.02) & 0.003 (IQR: 0.007) & 0.003 (IQR: 0.008) & 0.283 \\
\hline $\mathrm{CRP}(\mathrm{mg} / \mathrm{L} ;$ normal range $0-3$ ) & $46.29 \pm 59.72$ & $49.37 \pm 66.28$ & 0.233 \\
\hline
\end{tabular}

IQR: Interquartile range; RT-PCR: Real-time polymerase chain reaction; CRP: C-reactive protein; CT: Computed tomography; *: Chronic respiratory diseases includes chronic obstructive pulmonary disease and asthma. 
Table 2. Relationship between test positivity and incidence of alarm symptoms among patients with symptoms

\begin{tabular}{lccc}
\hline $\begin{array}{l}\text { Patients with any symptom } \\
\mathbf{n}=\mathbf{6 4 6}\end{array}$ & $\begin{array}{c}\text { Only RT-PCR(+) } \\
\mathbf{n = 5 6}\end{array}$ & $\begin{array}{c}\text { Only CT (+) } \\
\mathbf{n = 2 9 6}\end{array}$ & $\begin{array}{c}\text { Both RT-PCR and CT (+) } \\
\mathbf{n = 1 5 7}\end{array}$ \\
\hline $\begin{array}{l}\text { Alarm symptoms (\%) } \\
\quad \text { Fever }\end{array}$ & $29(51.8)^{*}$ & $172(58.1)$ & $106(67.5)$ \\
Cough & $22(39.3)$ & $200(67.6)^{*}$ & $84(53.5)$ \\
Dyspnea & $11(19.6)$ & $126(42.6)^{*}$ & $55(35.0)$ \\
\hline
\end{tabular}

$\mathrm{CT}$ : Computed tomography; RT-PCR: Real-time polymerase chain reaction; *: Those with significant relationship between test positivity and symptom presence has been marked.

Table 3. The performance of CT for COVID-19 infection with RT-PCR result as reference

\begin{tabular}{|c|c|c|c|c|c|}
\hline All patients, \% $(n=687)$ & $72.5(169 / 233)$ & $31.5(143 / 454)$ & $35.2(169 / 480)$ & $69.1(143 / 207)$ & $45.4(312 / 687)$ \\
\hline Patients with any symptom, $\%(n=646)$ & $73.7(157 / 213)$ & $31.6(137 / 433)$ & $34.6(157 / 453)$ & $70.9(137 / 193)$ & $45.5(294 / 646)$ \\
\hline Patients with contact history, $\%(n=109)$ & $65.3(49 / 75)$ & $44.1(15 / 34)$ & $72.1(49 / 68)$ & $36.5(15 / 41)$ & $58.7(64 / 109)$ \\
\hline
\end{tabular}

CT: Computed tomography; NPV: Negative predictive value; RT-PCR: Real-time polymerase chain reaction; PPV: Positive predictive value.

results $(92.9 \%)$ had at least one symptom. The rate of CT positive patients among patients with negative RT-PCR was 311/454 (68.5\%).

When we accept RT-PCR as a standard diagnostic method; CT's sensitivity was $72.5 \%(95 \% \mathrm{Cl}, 66-78 \%)$, specificity was $31.5 \%(95 \% \mathrm{Cl}, 27-36 \%)$, and accuracy was $45.5 \%$ (95\% Cl, 42-49\%) PPV and NPV for CT as diagnostic test were $35.2 \%$ and $69.1 \%$, respectively. PLR and NLR were 1.05 and 0.87 , respectively. According to symptom presence and contact history, CT's diagnostic performance is shown in Table 3. CT's specificity was $31.6 \%$ and $44.1 \%$, respectively, in the presence of at least one symptom and presence of contact history situations. However, there was no significant difference in $\mathrm{CT}$ positivity rates according to the presence of symptoms and contact history in the RT-PCR positive patient group $(p=0.189$ and $p=0.090$ ). The relationship between $C T$ positivity and symptoms was examined among patients with RT-PCR positive. Frequency of fever, cough, and dyspnea (alarm symptoms) was higher in patients with $\mathrm{CT}$ positive patients $(p=0.016)$.

\section{Laboratory Findings}

Laboratory findings of both RT-PCR and CT positive patients are summarized in Table 1. When laboratory findings regarding the relationship with mortality were examined, the neutrophil and lymphocyte counts of patients with exitus were significantly lower compared to other patients $(p=0.006$ and $p=0.000$, respectively). Median $D$ dimer, procalcitonin, troponin values, and mean $\mathrm{C}$-reactive protein
Table 4. Imaging findings of CT positive patients with COVID-19

\begin{tabular}{lccc}
\hline $\begin{array}{l}\text { Lung involvement } \\
\text { patients } \\
\text { n=480) }\end{array}$ & $\begin{array}{c}\text { CT (+) } \\
\text { and RT-PCR (+) } \\
\text { patients } \\
\text { (n=169) }\end{array}$ & Both CT & p \\
\hline Right (\%) & $114(23.8)$ & $22(13.0)$ & 0.000 \\
Left (\%) & $79(16.5)$ & $16(9.5)$ & 0.002 \\
Bilateral (\%) & $287(59.8)$ & $131(77.5)$ & 0.000 \\
Extent of lesions (\%) & & & \\
Focal & $194(40.4)$ & $60(35.5)$ & 0.106 \\
$\quad$ Multifocal & $230(47.9)$ & $77(45.6)$ & 0.447 \\
$\quad$ Diffuse & $56(11.7)$ & $32(18.9)$ & 0.000 \\
$\quad$ Peripheral distribution & $211(44.0)$ & $87(51.5)$ & 0.014 \\
Pattern of opacities (\%) & & & \\
Pure GGO & $230(47.9)$ & $91(53.8)$ & 0.055 \\
Complicated GGO & $74(15.4)$ & $32(18.9)$ & 0.116 \\
Consolidation & $61(12.7)$ & $9(5.3)$ & 0.000 \\
Mixed & $115(24.0)$ & $37(21.9)$ & 0.435 \\
Other findings (\%) & & & \\
Pleural effusion & $53(10.9)$ & $16(9.5)$ & 0.471 \\
Pulmonary nodules & $48(9.9)$ & $13(7.7)$ & 0.246 \\
Pulmonary fibrosis & $16(3.3)$ & $10(6.0)$ & 0.017 \\
Pleural thickening & $15(3.1)$ & $12(7.1)$ & 0.000 \\
Lymphadenopathy & $2(0.4)$ & $1(0.6)$ & 0.647 \\
Pulmonary emphysema & $4(0.8)$ & - & - \\
\hline
\end{tabular}

CT: Computed tomography; GGO: Ground glass opacity; RT-PCR: Real-time polymerase chain reaction.

(CRP) values in patients with exitus were also significantly higher. For each of these laboratory parameters, the calculated $P$ value was 0.000 . 


\section{CT Findings}

Imaging characteristics of 480 patients with CT positive are specified in Table 4. Most common CT pattern was pure GGO 230/480 (47.9\%). The least common pattern was consolidation pattern $61 / 480$ (12.7\%). The relationship between RTPCR positivity and CT findings was examined in CT positive patients. The incidence of consolidation pattern in RT-PCR positive patients was determined to be significantly lower $(p=0.000)$. Again, the frequency of GGO-related findings in RT-PCR positive patients was significantly higher ( $p=0.002)$. When other variables were examined, it was found that there was only a correlation between mortality and CT findings. Mixed pattern's incidence was significantly higher in patients with exitus ( $p=0.001$ ). The incidence of GGO-related findings in patients with exitus was found to be significantly lower $(p=0.000)$.

\section{Patient Outcomes}

It was observed that among the patients 572 (83.3\%) were followed in service and 115 (16.7\%) were followed in intensive care. In both RT-PCR and CT positive patients, the frequency of intensive care follow-up was significantly higher $(p=0.005)$. The average length of stay in hospital was 7.8 \pm 4.3 days. The length of stay in hospital for both RT-PCR and CT positive patients had increased significantly (mean difference: $-4.2,95 \% \mathrm{Cl}:-4.9,-3.4)(p=0.000)$. CFR was $6 \%$ (41 patients) for all patients. CFR was significantly higher in both RT-PCR and CT positive patients $(p=0.01)$. No significant relationship was found between RT-PCR positivity alone or CT positivity and hospitalization and CFR data alone.

According to logistic regression analysis, independent factors that may have an impact on mortality were examined. Male sex ( $p=0.037$; OR: $0.385 ; 95 \% \mathrm{Cl}, 0.157-0.943)$, higher age ( $p=0.000 ;$ OR: 1,$068 ; 95 \% \mathrm{Cl}, 1,031-1.106)$, and presence of any comorbidity ( $\mathrm{p}=0.008$; OR: 5,$374 ; 95 \% \mathrm{Cl}$, $1,539-7.618$ ) were and mortality were found to be associated with the mortality outcome.

\section{Discussion}

The COVID-19 pandemic process is a period that requires a thorough evaluation of each case alone. In this study, we found that the use of RT-PCR, CT, and patient's clinical data together was main weapon in both diagnosis and monitoring in the battle with this new viral factor. Hospitalization time, intensive care follow-up rate, and CFR had increased significantly in both RT-PCR and CT positive patients. Furthermore, when we examine the independent factors; we found that higher age, male gender, and presence of any comorbidity were effective on mortality.

In line with similar literature; more than half of the patients in our study were males (62.4\%), the average age was around $50^{[13,14]}$. Proportion of patients with at least one comorbid disease was (49.9\%) similar to those in Wang et al. and Chen et al.'s studies. The most common comorbid disease we encountered in our study was hypertension as mentioned in the literature ${ }^{[7,13]}$. The most common complaints of our patients were fever, cough, and dyspnea ${ }^{[15]}$. In addition, when we examine laboratory parameters, the number of neutrophil and leukocytes of cases that end with mortality in accordance with the literature is low; CRP, procalcitonin, troponin, and D dimer values are significantly higher ${ }^{[16]}$.

Although the standard test is considered to be RT-PCR in the diagnosis of COVID-19, it is known that in some cases falsenegative results may be experienced in the early period ${ }^{[17]}$. In such cases, thoracic CT has become a highly useful and accepted option ${ }^{[18]}$. In our study, when we considered RTPCR as a reference test, we determined CT's sensitivity to be $72.5 \%$. This was similar to the work He et al. ${ }^{[19]}$ including 82 patients. In our study, the specificity (31.5\%) and accuracy $(45.5 \%)$ values we have identified for $\mathrm{CT}$ values were similar to the study by Ai et al. ${ }^{[10]}$ in which they examined 1014 patients. We also found that in the presence of clinical conditions such as symptom and contact history, CT's specificity, and accuracy has increased. This has shown us that every piece of data we obtain about patients from the moment of application functions as part of a puzzle during the diagnostic process.

The evaluation of patients who are considered as clinically possible cases of COVID-19 with both RT-PCR and CT from the moment of application, the "comprehensive screening strategy," has been effectively applied in China since the beginning of the outbreak. Similar to the China, Turkish Ministry of Health has made it mandatory to implement an evaluation based on detailed examination of patients from the first moment, with the COVID-19 algorithm. In our study, we evaluated the power of this application in detail. There was a significant relationship between the positivity of both diagnostic examinations and CFR, intensive care hospitalization, and increased length of stay in hospital. We found that RT-PCR positivity alone and CT positivity alone were insufficient to give clues about the poor clinical outcomes of patients. This means that while diagnostic tests are insufficient to show bad prognosis individually, they increase the diagnostic value of each other when used together. Based 
on this information, we can say that the COVID-19 pandemic showed us that clinical management and diagnosis of these patients is not just a one-way process.

The most common radiological finding we encountered in our study was pure GGO pattern. Most of our cases had bilateral lung involvement, and lesions had predominantly multifocal and peripheral distribution. These radiological findings we identified were consistent with the findings seen in COVID-19 cases mentioned in the literature ${ }^{[20]}$. In our RT-PCR positive patients, the frequency of GGO-related radiological findings increased and the frequency of consolidation patterns decreased. In their study, Shi et al. ${ }^{[6]}$ stated that in cases where the disease is progressive, GGOrelated findings decrease and mixed patterns increase. In our study, the dominant lesion type mixed pattern was in the CT of patients with exitus, and the incidence of GGOrelated lesion was quite low in these cases.

Although the patient data from the early period of the pandemic process in our country were examined in our study, the CFR of our hospitalized patients was $6 \%$. In early studies conducted in China, the CFR was around $15 \%$, while subsequent studies indicated that these rates were distributed as $4.3 \%$ and $11.0 \%$ and even decreased to $2.3 \%{ }^{[21]}$. Although the CFR we identified in our study was quite low compared to the initial studies in the literature, it was in line with the Global mortality rate of $5.7 \%,{ }^{[22,23]}$ which the WHO has stated about March 2020. In addition, in the logistic regression analysis we do, in accordance with the literature; we also had the opportunity to examine this crowded patient population that the presence of advanced age, male sex, and comorbid disease was effective on mortality ${ }^{[24]}$.

The low age of cases in our study can be associated with our low mortality rates. However, considering that the frequency of comorbid disease in our cases is quite high, we believe that low CFR may be associated with patient care quality and the compliance of all health centers with a common algorithm. The COVID-19 case management algorithm in our country includes anamnesis, clinical data, physical examination, and RT-PCR and CT findings. With this algorithm, the multifaceted patient approach across the country has been transformed into a standard procedure. In the early period of the pandemic, algorithms were constantly updated, providing a dynamic data stream, ensuring that all clinicians were constantly informed.

\section{Limitations}

The most basic limitation of our study is that it does not include details in treatment and post-hospitalization mon- itoring, as we only evaluate the emergency room admission and examination process. Only intensive care/service hospitalization rates, length of stay in hospital and CFR was examined for patient termination. However, since the application, we believe that we have conducted a thorough research by evaluating anamnesis, clinical findings and vital findings, laboratory examinations, and CT results for patients. Another limitation of this study is that only patients who applied to the emergency department were included in the study. More comprehensive patient data could be obtained with a study that includes patients who apply to pandemic policlinics.

\section{Conclusion}

As with all mass events, the emergency services in our country have been the first point of application for possible COVID-19 cases in the pandemic process. The combination of anamnesis, clinical, and laboratory data and CT in emergency departments has played a very important role in early detection and isolation of possible COVID-19 cases in the process until RT-PCR results are obtained. We believe that the multifaceted patient evaluation and CT interpretation skills of physicians working in emergency departments are an effective factor in the fight against COVID-19.

Ethics Committee Approval: All procedures performed in studies involving human participants were in accordance with the ethical standards of the institutional and/or national research committee and with the 1964 Declaration of Helsinki and its later amendments or comparable ethical standards. The ethics committee approval for the study was taken from the ethics committee of Ataturk University Faculty of Medicine (date: 23.01.2020, number: 75).

Peer-review: Externally peer-reviewed.

Authorship Contributions: Concept: I.A., A.O.K., S.D., D.T., B.A., A.A.; Design: I.A., S.D., A.O.K., S.A., D.T., Z.Ç.; Data Collection or Processing: I.A., A.O.K., S.A., A.A., B.A.; Analysis or Interpretation: I.A., A.O.K., S.D., D.T., S.A., A.A., B.A., Z.Ç.; Literature Search: I.A., S.D., S.A.; Writing: I.A., S.D., S.A., A.O.K., D.T., A.A.

Conflict of Interest: None declared.

Financial Disclosure: The authors declared that this study received no financial support.

\section{References}

1. Ye Z, Zhang $Y$, Wang $Y$, Huang $Z$, Song B. Chest CT manifestations of new coronavirus disease 2019 (COVID-19): A pictorial review. Eur Radiol 2020;30:4381-9. [CrossRef]

2. World Health Organization Coronavirus Disease 2019 (COVID-19) Situation Report 39. Geneva:World Health Organization; 2020. Available at: https://www.who.int/docs/default- 
source/coronaviruse/situation-reports/20200228-sitrep-39covid-19.pdf?sfvrsn=5bbf3e7d_4.v Accessed on February 28, 2020.

3. Ministry of Health General Directorate of Public Health, Covid-19 (Sars-Cov2 Infection) Directory, Coronavirus Scientific Advisory Board, Turkey. Turkey: Ministry of Health General Directorate of Public Health; 2020.

4. Wang W, Xu Y, Gao R, Lu R, Han K, Wu G, et al. Detection of SARS-CoV-2 in different types of clinical specimens. JAMA 2020;323:1843-4. [CrossRef]

5. Pan Y, Guan H, Zhou S, Wang Y, Li Q, Zhu T, et al. Initial CT findings and temporal changes in patients with the novel coronavirus pneumonia (2019-nCoV): A study of 63 patients in Wuhan, China. Eur Radiol 2020;30:3306-9. [CrossRef]

6. Shi H, Han X, Jiang N, Cao Y, Alwalid O, Gu J, et al. Radiological findings from 81 patients with COVID-19 pneumonia in Wuhan, China: A descriptive study. Lancet Infect Dis 2020;20:425-34. [CrossRef]

7. Wang D, Hu B, Hu C, Zhu F, Liu X, Zhang J, et al. Clinical characteristics of 138 hospitalized patients with 2019 novel coronavirus-infected pneumonia in Wuhan, China. JAMA 2020;323:1061-9. [CrossRef]

8. Chung M, Bernheim A, Mei X, Zhang N, Huang M, Zeng X, et al. CT imaging features of 2019 novel coronavirus (2019-nCoV). Radiology 2020;295:202-7. [CrossRef]

9. Majidi $H$, Niksolat F. Chest $C T$ in patients suspected of COVID-19 infection: A reliable alternative for RT-PCR. Am J Emerg Med 2020;38:2730-2. [CrossRef]

10. Ai T, Yang Z, Hou H, Zhan C, Chen C, Lv W, et al. Correlation of chest CT and RT-PCR testing for coronavirus disease 2019 (COVID-19) in China: A report of 1014 cases. Radiology 2020;296:E32-40. [CrossRef]

11. Republic of Turkey Ministry of Health. Updated COVID-19 National Guideline of Health Ministry of Turkey. Ankara, Turkey: Republic of Turkey Ministry of Health; 2020.

12. Song F, Shi N, Shan F, Zhang Z, Shen J, Lu H, et al. Emerging 2019 novel coronavirus (2019-nCoV) pneumonia. Radiology 2020;295:210-7. [CrossRef]

13. Chen N, Zhou M, Dong X, Qu J, Gong F, Han Y, et al. Epidemiological and clinical characteristics of 99 cases of 2019 novel coronavirus pneumonia in Wuhan, China: A descriptive study.
Lancet 2020;395:507-13. [CrossRef]

14. Huang C, Wang Y, Li X, Ren L, Zhao J, Hu Y, et al. Clinical features of patients infected with 2019 novel coronavirus in Wuhan, China. Lancet 2020;395:497-506. [CrossRef]

15. Grasselli G, Zangrillo A, Zanella A, Antonelli M, Cabrini L, Castelli A, et al. Baseline characteristics and outcomes of 1591 patients infected with SARS-CoV-2 admitted to ICUs of the Lombardy region, Italy. JAMA 2020;323:1574-81. [CrossRef]

16. Zhang JJ, Dong X, Cao YY, Yuan YD, Yang YB, Yan YQ, et al. Clinical characteristics of 140 patients infected with SARS-CoV-2 in Wuhan, China. Allergy 2020;75:1730-41. [CrossRef]

17. Chen D, Jiang X, Hong Y, Wen Z, Wei S, Peng G, et al. Can chest $C T$ features distinguish patients with negative from those with positive initial RT-PCR results for coronavirus disease (COVID-19)? AJR Am J Roentgenol 2021;216:66-70. [CrossRef]

18. Abbasi-Oshaghi E, Mirzaei F, Farahani F, Khodadadi I, Tayebinia $\mathrm{H}$. Diagnosis and treatment of coronavirus disease 2019 (COVID-19): Laboratory, PCR, and chest CT imaging findings. Int J Surg 2020;79:143-53. [CrossRef]

19. He JL, Luo L, Luo ZD, Lyu JX, Ng MY, Shen XP, et al. Diagnostic performance between CT and initial real-time RT-PCR for clinically suspected 2019 coronavirus disease (COVID-19) patients outside Wuhan, China. Respir Med 2020;168:105980. [CrossRef]

20. Hu L, Wang C. Radiological role in the detection, diagnosis and monitoring for the coronavirus disease 2019 (COVID-19). Eur Rev Med Pharmacol Sci 2020;24:4523-8.

21. Rajgor DD, Lee MH, Archuleta S, Bagdasarian N, Quek SC. The many estimates of the COVID-19 case fatality rate. Lancet Infect Dis 2020;20:776-7. [CrossRef]

22. Rodriguez-Morales AJ, Cardona-Ospina JA, Gutierrez-Ocampo E, Villamizar-Pena R, Holguin-Rivera Y, Escalera-Antezana JP, et al. Clinical, laboratory and imaging features of COVID-19: A systematic review and meta-analysis. Travel Med Infect Dis 2020;34:101623. [CrossRef]

23. Baud D, Qi X, Nielsen-Saines K, Musso D, Pomar L, Favre G. Real estimates of mortality following COVID-19 infection. Lancet Infect Dis 2020;20:773. [CrossRef]

24. Nikpouraghdam M, Farahani AJ, Alishiri G, Heydari S, Ebrahimnia M, Samadinia $H$, et al. Epidemiological characteristics of coronavirus disease 2019 (COVID-19) patients in IRAN: A single center study. J Clin Virol 2020;127:104378. [CrossRef] 
Appendix 1.

\section{GUIDE ON POSSIBLE COVID-19 CASE INQUIRY FOR OUTPATIENTS}

Triage is performed by a healthcare professional dressed in accordance with the COVID-19 Case Algorithm (gown, medical mask, face shield, or goggles).
Do you have a fever or a history of fever?
Yyes $\bigcirc$ No
Do you have a cough?
Yes $\bigcirc$ No
Do you have difficulty in breathing or respiratory distress?
Yes $\bigcirc$ No
If the answer to any of the above questions is YES, the
PATIENT WEARS A MASK and is referred to the area dedicated to COVID-19.

If the answer to all of the above questions is NO, the patient is asked the following questions.

Have you been abroad in the past 14 days?<smiles>O[Mg][Nb]</smiles>

Has a family member come from abroad in the last 14 days?<smiles>O[Mg][Nb]</smiles>

Has any of your relatives been hospitalized for respiratory disease in the past 14 days?<smiles></smiles>

Has any of your relatives been diagnosed with COVID-19 disease in the past 14 days?
Yes
No

If the answer to any of the questions above is YES, the PATIENT WEARS A MASK because of the risk of COVID19 and referred to the area dedicated to COVID-19.

\section{If the answer to all of the above questions is NO, patient is considered \\ to be at low risk for COVID-19 and referred to the relevant \\ department for consideration in line with his complaint.}


Appendix 2.

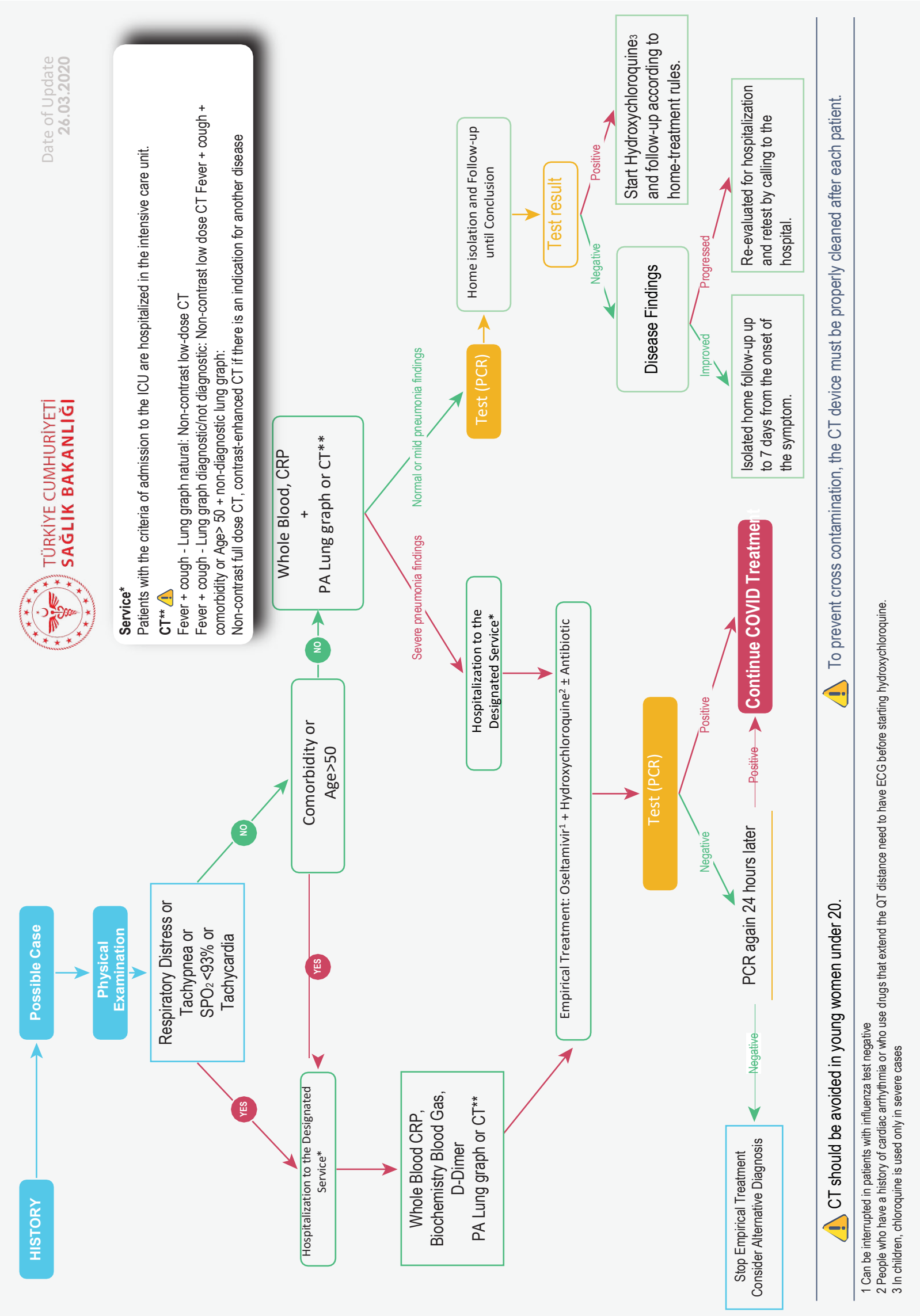

